\title{
BMJ Open Gout and hearing impairment in the elderly: a retrospective cohort study using the US Medicare claims data
}

\author{
Jasvinder A Singh, ${ }^{1,2,3}$ John D Cleveland ${ }^{2}$
}

To cite: Singh JA, Cleveland JD. Gout and hearing impairment in the elderly: a retrospective cohort study using the US Medicare claims data. BMJ Open 2018;8:e022854. doi:10.1136/ bmjopen-2018-022854

- Prepublication history for this paper is available online. To view these files, please visit the journal online (http://dx.doi. org/10.1136/bmjopen-2018022854).

Received 9 March 2018 Revised 25 June 2018 Accepted 26 July 2018

D) Check for updates

(C) Author(s) (or their employer(s)) 2018. Re-use permitted under CC BY-NC. No commercial re-use. See rights and permissions. Published by BMJ.

${ }^{1}$ Medicine Service, VA Medical Center, Birmingham, Alabama, USA

${ }^{2}$ Department of Medicine,

School of Medicine, University of Alabama, Birmingham, Alabama, USA

${ }^{3}$ Division of Epidemiology, School of Public Health, University of Alabama, Birmingham, Alabama, USA

Correspondence to Dr Jasvinder A Singh; jasvinder.md@gmail.com

\begin{abstract}
Objectives To evaluate whether gout is associated with a higher risk of hearing loss in older adults.

Design Retrospective cohort study.

Setting USA.

Participants $5 \%$ random sample of US Medicare claims 2006-2012, representative of US adults aged 65 years or older.

Primary and secondary outcomes Incident (new) hearing loss identified by the presence of at least two claims at least 4 weeks apart with an International Classification of Diseases, Ninth Revision, 389.xx, with no respective claim in the baseline 1 -year observation period. Results Among the 1.71 million eligible people, 89409 developed incident hearing impairment. The crude incidence rates of incident hearing impairment in people with versus without gout were 16.9 vs. 8.7 per 1000 person-years. Using Cox regression analyses adjusted for demographics, medical comorbidity and common cardiovascular and gout medications, we found that gout was associated with a significantly higher HR of incident hearing impairment, HR was $1.44(95 \% \mathrm{Cl} 1.40$ to 1.49 , $p<0.0001)$. Findings were confirmed in sensitivity analyses that substituted continuous Charlson-Romano Index with categorical variable or all comorbidities and additionally cardiovascular risk factors, with minimal attenuation of HR. Conclusions Gout is associated with a higher risk of development of hearing loss in older adults. Future studies need to assess the underlying mechanisms of this association.
\end{abstract}

\section{INTRODUCTION}

Hearing loss is a common problem in the ageing population. The 2005-5006 National Health and Nutritional Examination Survey (NHANES) data showed that more than two-thirds of US adults aged 70 years or older had hearing loss, ${ }^{1}$ while another population-based study found a prevalence of $46 \%$ in US adults aged $48-92$ years. $^{2}$ People with hearing loss continue to have further decline in hearing. ${ }^{3}$ Hearing loss is associated with self-reported communication difficulties, difficulty with activities of daily living (ADLs) and instrumental ADLs and a lower quality of life. ${ }^{4-6}$ Hearing loss is associated with a higher risk of incident

\section{Strengths and limitations of this study}

- Study findings are only generalisable to adults aged 65 years or older.

- We used the diagnostic codes for both the outcome and the exposure, which puts results at the risk of misclassification bias, which we estimate would bias our estimates towards null.

- Lack of laboratory measures, including serum urate, or markers of inflammation or oxidative stress limited us from assessing whether they are the underlying mechanisms of this association.

- Despite controlling for several potential confounders, residual confounding is possible, due to an observational study design.

- Our study strengths were that we included a representative large sample of US adults aged 65 years or older, an adequate number of events occurred and our study findings were robust in multiple sensitivity models.

dementia $^{7}$; sudden hearing loss is associated with a higher risk of stroke. ${ }^{8}$ Thus, hearing loss, with its consequences and long-term individual and societal burden, is a significant public health problem.

A recent study using the NHANES data found that older age, male sex and white race were strongly associated with hearing loss after multivariate adjustment ${ }^{1}$; cardiovascular disease, loud noise, diabetes and smoking were also associated. ${ }^{910}$ In mouse cochlea, increased oxidative stress and inflammation may trigger cell death pathways. ${ }^{11}$ Other studies have also shown that oxidative stress, inflammation and autophagic stress can potentiate noise-induced or age-related hearing loss. ${ }^{11-14}$

Another condition with risk factors similar to hearing loss (older age, male sex, cardiovascular disease) is gout, the most common inflammatory arthritis in adults. The hallmarks of gout are hyperuricaemia and urate crystal formation that are associated with inflammation ${ }^{15} \quad 16$ and oxidative stress, ${ }^{17-21}$ pathological processes which are also 
implicated in the pathogenesis of hearing loss. ${ }^{11-14}$ Based on this similarity in pathogenesis, we hypothesised that gout in the elderly will be independently associated with a higher risk of new-onset hearing loss after adjusting for known risk factors, and that this association will vary by important biological variables, such as age, sex and race. Our objective was to address these questions.

\section{METHODS}

\section{Data sources and study sample}

We used the 5\% Medicare claims data from 2006 to 2012. People were eligible for this study if they were Medicare fee-for-service recipients with part A and/or B, but not Medicare Advantage plan (part C; claims incomplete) and a valid US mailing address.

\section{Predictor of interest}

The predictor of interest was existing gout, identified by the occurrence of two International Classification of Diseases, Ninth Revision (ICD-9-CM) codes for 274.xx, on two claims at least 4 weeks apart, an approach shown to be valid in observational studies with specificity and sensitivity of $\geq 90 \%{ }^{22}$

\section{Independent variable/outcome of interest}

The outcome we were interested in was incident (new) hearing impairment, identified by the presence of at least two claims at least 4 weeks apart with an ICD-9-CM, 389.xx, ${ }^{23}$ and its absence in the baseline 1-year period. We included patient demographics, age, sex and race, based on the data obtained from the Medicare denominator file and the beneficiary summary file. We assessed medical comorbidity using the Charlson-Romano Comorbidity Index which is a validated measure of comorbidity, developed for claims data. ${ }^{24}$ It was obtained from inpatient and outpatient Medicare claims and treated as a continuous variable (main analysis). We used the Medicare part D prescription claims file to assess the use of common cardiovascular drugs (statins, beta-blockers, diuretics and ACE inhibitors) and drugs for gout (allopurinol, febuxostat), as surrogates for the severity (and the presence) of the underlying conditions which they were used to treat.

\section{Patient and public involvement}

The development of the research question was informed by several patients with gout seen at the University of Alabama at Birmingham (UAB) General Rheumatology Clinic and the UAB Gout Clinic who asked us about the possible link of gout and their chronic condition(s), including hearing loss. This prompted us to perform this study. Patients were not involved in study design or conduct. Administrative dataset does not contain identifiable information for patients, and therefore, patients can not be contacted for dissemination of study findings. The publication of this manuscript is our planned dissemination of the findings to the public.

\section{Statistical analyses}

We calculated crude incidence rates for the occurrence of incident hearing impairment in patients with versus without gout at baseline. Characteristics of people with versus without hearing impairment were compared using Student's t-test or $\chi^{2}$ test, as appropriate. We used multivariable-adjusted Cox proportional hazard model to assess whether gout diagnosis at baseline was associated with a new diagnosis of hearing impairment during the follow-up, while adjusting for demographics, comorbidity and common medications (model 1). Sensitivity analyses modelled Charlson-Romano Index in categories (model 2) or as individual comorbidities plus hyperlipidemia, hypertension and coronary artery disease, ie, cardiovascular risk factors (model 3). Subgroup analyses were done to assess if gout's association with hearing impairment varied by age, gender, race and tobacco use disorder.

\section{RESULTS}

Of the 1.71 million people in our cohort, 89409 developed incident hearing impairment (table 1). Compared with the people without, people with hearing impairment were older, less likely to be black and had higher medical comorbidity (table 1). The crude incidence rates of incident hearing impairment in people with versus without gout were 16.9 vs 8.7 per 1000 person-years.

In multivariable-adjusted analyses, we found that gout was associated with a significantly increased HR of new-onset hearing impairment, HR was 1.44 (95\% CI 1.40 to 1.49 ), confirmed with minimal attenuation of HR in sensitivity analyses (models 2 and 3; table 2).

In subgroup analyses, the HR of gout with hearing loss was similar across age, gender and race subgroups, although the interaction term was statistically significant for age, gender and race ( $\mathrm{p}$ values $<0.0001,0.001$ and 0.05 , respectively; table 3 ). Gout was significantly associated with hearing impairment in people without tobacco use disorder and in those with tobacco use disorder, but the interaction term was statistically not significant (table 3 ).

\section{DISCUSSION}

In this study of adults aged 65 years or older, we found an independent association of gout with a $44 \%$ higher risk of new hearing impairment after adjusting for demographics, medical comorbidities and the commonly used medications for cardiovascular disease and gout. These findings were robust across multiple sensitivity analyses.

Our study has several limitations. Our study findings are only generalisable to adults aged 65 years or older. We used the diagnostic codes for outcome and exposure, which are subject to misclassification bias, which would bias our estimates towards null and indicates that our estimates were conservative. However, we used valid algorithms for identifying people with hearing loss and gout. ${ }^{22}{ }^{23}$ We did not have access to laboratory measures, including serum urate, or markers of inflammation (eg, 
Table 1 Demographic and clinical characteristics of people with hearing impairment

\begin{tabular}{|c|c|c|c|c|}
\hline & \multirow[b]{2}{*}{ Entire cohort } & \multicolumn{2}{|c|}{ Hearing impairment during the follow-up } & \multirow[b]{2}{*}{$P$ values } \\
\hline & & No & Yes & \\
\hline Total, N & $1716438^{*}$ & 1627029 & 89409 & \\
\hline Age, mean (SD) & $75.3(7.6)$ & $75.2(7.6)$ & $76.5(7.3)$ & $<0.0001$ \\
\hline Gender, N (\%) & & & & $<0.0001$ \\
\hline Male & 728050 (42.4) & $691144(42.5)$ & 36906 (41.3) & \\
\hline Female & $988388(57.6)$ & 935885 (57.5) & $52503(58.7)$ & \\
\hline Race/ethnicity, N (\%) & & & & $<0.0001$ \\
\hline White & $1477659(86.1)$ & 1397909 (85.9) & $79750(89.2)$ & \\
\hline Black & $141798(8.3)$ & $137200(8.4)$ & $4598(5.1)$ & \\
\hline Other/unknown & $96981(5.7)$ & $91920(5.6)$ & $5061(5.7)$ & \\
\hline $\begin{array}{l}\text { Charlson-Romano comorbidity score, } \\
\text { mean (SD) }\end{array}$ & $1.60(2.39)$ & $1.59(2.40)$ & $1.75(2.23)$ & $<0.0001$ \\
\hline $\begin{array}{l}\text { Charlson-Romano comorbidity score } \\
\text { categories, } \mathrm{N}(\%)\end{array}$ & & & & $<0.0001$ \\
\hline 0 & 905578 (52.8\%) & 866797 (53.3\%) & $38781(43.4 \%)$ & \\
\hline 1 & $172125(10.0 \%)$ & $160939(9.9 \%)$ & $11186(12.5 \%)$ & \\
\hline$\geq 2$ & $638735(37.2 \%)$ & $599293(36.8 \%)$ & $39442(44.1 \%)$ & \\
\hline \multicolumn{5}{|l|}{ Charlson-Romano Comorbidities, N (\%) } \\
\hline Myocardial infarction & $68199(4.0)$ & $64294(4.0)$ & 3905 (4.4) & $<0.0001$ \\
\hline Heart failure & $201363(11.7)$ & $190652(11.7)$ & $10711(12.0)$ & 0.018 \\
\hline Peripheral vascular disease & $166866(9.7)$ & $156169(9.6)$ & $10697(12.0)$ & $<0.0001$ \\
\hline Cerebrovascular disease & $166659(9.7)$ & $156026(9.6)$ & $10633(11.9)$ & $<0.0001$ \\
\hline Dementia & $77454(4.5)$ & $74434(4.6)$ & 3020 (3.4) & $<0.0001$ \\
\hline Chronic pulmonary disease & $267689(15.6)$ & 251447 (15.5) & $16242(18.2)$ & $<0.0001$ \\
\hline Connective tissue disease & $47429(2.8)$ & $43887(2.7)$ & $3542(4.0)$ & $<0.0001$ \\
\hline Peptic ulcer disease & $32397(1.9)$ & $30202(1.9)$ & $2195(2.5)$ & $<0.0001$ \\
\hline Mild liver disease & $8440(0.49)$ & $7957(0.49)$ & $483(0.54)$ & 0.033 \\
\hline Diabetes & $317322(18.5)$ & $299093(18.4)$ & $18229(20.4)$ & $<0.0001$ \\
\hline Diabetes with end organ damage & $93630(5.5)$ & $87895(5.4)$ & $5735(6.4)$ & $<0.0001$ \\
\hline Hemiplegia & $14238(0.83)$ & $13515(0.83)$ & $723(0.81)$ & 0.48 \\
\hline Renal failure/disease & $59136(3.4)$ & $56187(3.5)$ & 2949 (3.3) & 0.013 \\
\hline Any tumour, leukaemia or lymphoma & $171928(10.0)$ & $160323(9.9)$ & 11605 (13.0) & $<0.0001$ \\
\hline Moderate or severe liver disease & $1985(0.12)$ & $1905(0.12)$ & $80(0.09)$ & 0.018 \\
\hline Metastatic cancer & $17831(1.0)$ & $17109(1.1)$ & $722(0.81)$ & $<0.0001$ \\
\hline AIDS & $546(0.03)$ & $520(0.03)$ & $26(0.03)$ & 0.64 \\
\hline Hypertension & 826095 (48.1) & $771614(47.4)$ & $54481(60.9)$ & $<0.0001$ \\
\hline Hyperlipidaemia & 594495 (34.6) & 552056 (33.9) & 42439 (47.5) & $<0.0001$ \\
\hline Coronary artery disease & 300512 (17.5) & 280016 (17.2) & $20496(22.9)$ & $<0.0001$ \\
\hline Obesity & $35772(2.1)$ & $33622(2.1)$ & $2150(2.4)$ & $<0.0001$ \\
\hline
\end{tabular}

Bold values represent statistically significant difference with a $p$-value of $<0.05$.

${ }^{*}$ Cohort selected for study of incident hearing loss that had a baseline period of 365 days without a diagnosis of hearing loss.

C reactive protein) or oxidative stress. Therefore, we could not assess whether these mechanisms underlie the noted association between gout and hearing loss. Due to an observational study design, residual confounding from unmeasured variables (prior noise exposure or the use of ototoxic drugs) is possible, despite the fact that we controlled for several potential confounders. We examined additional outcomes in other related papers but did not make any adjustment for multiple testing in this analysis; however, even after correction for 15 hypothesis 


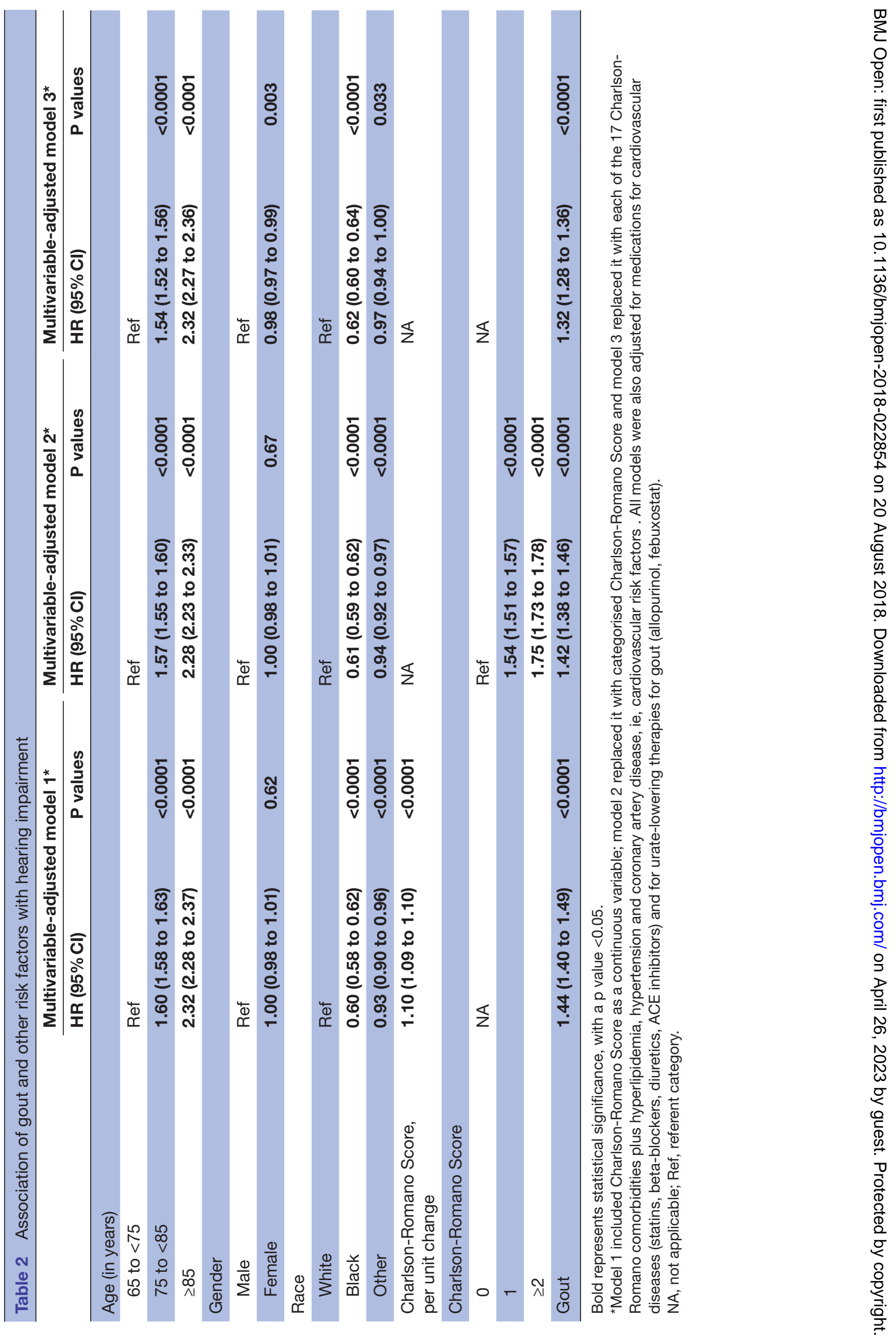


Table 3 Association of gout with hearing impairment, in predefined subgroups by race, gender, age and tobacco use disorder

\begin{tabular}{|c|c|c|c|c|c|c|}
\hline & \multicolumn{2}{|c|}{ Multivariable-adjusted model 1} & \multicolumn{2}{|c|}{ Multivariable-adjusted model 1} & \multicolumn{2}{|c|}{ Multivariable-adjusted model 1} \\
\hline & HR $(95 \% \mathrm{Cl})$ & $P$ values & HR $(95 \% \mathrm{Cl})$ & $P$ values & HR (95\% Cl) & $P$ values \\
\hline & $65-75$ years & & $75-85$ years & & $>85$ years & \\
\hline Gout & Female & & Male & & & \\
\hline Gout & 1.37 (1.31 to 1.44$)$ & $<0.0001$ & $1.50(1.44$ to 1.56$)$ & $<0.0001$ & & \\
\hline \multirow[t]{2}{*}{ Gout } & 1.56 (1.40 to 1.73$)$ & $<0.0001$ & $1.43(1.38$ to 1.48$)$ & $<0.0001$ & $1.53(1.36$ to 1.73$)$ & $<0.0001$ \\
\hline & No tobacco use dis & & Tobacco use disoro & & & \\
\hline Gout & $1.44(1.40$ to 1.49$)$ & $<0.0001$ & $1.48(1.12$ to 1.96$)$ & 0.006 & & \\
\hline
\end{tabular}

Agexgout $p$ value $<0.0001$

Sex×gout $p$ value 0.001

RacexGout $p$ value 0.05 .

Smoking $\times$ gout $p$ value 0.93

HRs that are significant with $p$ value $<0.05$ are in bold.

testing, the Bonferroni corrected p value would be 0.003 which was higher than the $\mathrm{p}$ value reported in our study. This means that even when corrected for multiple testings, the current study findings would still be significant. It is possible that a longer baseline period with no diagnosis would further increase the possibility of not missing the baseline diagnosis of hearing loss; however, this would lead to smaller cohort for observation of incident hearing loss cases and a shorter observation period. Weighing pros and cons, we chose to keep baseline period at 12 months which is standard for Medicare database studies (12-18 months).

Our study strengths were the inclusion of a representative sample of US adults aged 65 years or older, a large sample with an adequate number of events, and the robustness of our study findings.

To our knowledge, this study is among the first to describe an association of gout with hearing loss in older adults. However, other related studies of hyperuricaemia have shown similar findings. In a recent Korean study, a higher serum urate (sUA) was significantly associated with new age-related hearing loss in multivariable-adjusted analyses in adults 40 years or older. ${ }^{25}$ The previous study examined the association of hyperuricaemia with hearing loss and recruited people during a regular health check-up at a medical centre, as opposed to our study which examined the association of gout with hearing loss, and assessed all Americans aged 65 years or older. While the use of audiometry and the assessment of serum urate is an advantage with the previous study, the inclusion of a large representative study sample and the adjustment for common medication use and medical comorbidities is a strength of our study. Our study results are not directly comparable with those from the previous study, since the risk factors, while somewhat related, are not the same.

A recent case report of conductive hearing loss due to urate deposits in the middle ear likely illustrates the pathogenic role of urate crystals in hearing loss in the most severe forms of gout. ${ }^{26}$ Our finding of the $32 \%-44 \%$ increased hazards of hearing loss associated with the diagnosis of gout must be interpreted in the proper clinical context. An early recognition of hearing loss related to the presence of gout may have a meaningful clinical implication. Although the increased risk is not twofold or threefold higher (which is not an uncommon finding in epidemiological studies for a polygenic disease), it seems clinically meaningful. Screening and diagnosis of hearing loss is relatively inexpensive, and the economic impact of hearing loss is large, estimated at US $\$ 750$ billion lost each year globally. ${ }^{27}$ Hearing loss has a significant impact on quality of life, both physical and mental/ emotional health, and a higher severity of hearing loss is associated with larger deficits in quality of life $\mathrm{e}^{4}$; it is also associated with cognitive decline. ${ }^{28}$ Hearing aids not only improve the hearing, but may also protect against cognitive impairment and disability, improving quality of life of aged people. ${ }^{6}$ With an increasing emphasis on prevention of hearing loss, ${ }^{29}$ recognition of novel risk factors, such as gout, pave the way for an early diagnosis and potentially preventing more severe hearing loss in an ageing population, and decreasing the overall associated costs and disability.

Gout is a chronic inflammatory arthritis characterised by hyperuricaemia and urate crystal formation which subsequently lead to inflammation ${ }^{15} 16$ and oxidative stress. ${ }^{17-21}$ These processes could be one of the potential explanations of the association. Levels of serum malondialdehyde (MDA), a marker of lipid peroxidation, were significantly higher and antioxidant enzymes, erythrocyte superoxide dismutase (SOD) and erythrocyte catalase levels were lower in patients with gout. ${ }^{18}$ Treatment with allopurinol, a xanthine oxidase inhibitor that lowers serum urate, decreased serum MDA and increased erythrocyte SOD and catalase within 3 months of treatment. ${ }^{18}$ The use of febuxostat, another xanthine oxidase inhibitor urate-lowering drug, was associated with a reduction in the level 
of derivatives of reactive oxygen metabolites. ${ }^{30}$ Recurrent acute gout attacks are caused by urate-crystal-induced recruitment of leucocytes, activation of the complement system and the NALP3 inflammasome, leading to activation and extracellular release of interleukin (IL)-1ß and IL-18 and downstream effects of inflammation. ${ }^{1531}$

Both inflammation and increased oxidative stress are also implicated in the pathogenesis of hearing loss. ${ }^{11-14} 32$ In a mouse model of age-related hearing loss, the cochlear levels of MDA (a lipid peroxidation product) were significantly higher, proinflammatory cytokine (tumor necrosis factor (TNF)-alpha, IL-1b) levels were higher ${ }^{11}$ and mitochondrial DNA damage more frequent, compared with mice resistant to hearing loss. ${ }^{11}$ In a mouse model of age-related hearing loss with an early decline of the endocochlear potential due to severe mitochondrial and vascular pathologies in the cochlear stria vascularis, pathological alterations in antioxidant and nutrient and energy-sensing pathways (mammalian target of rapamycin (mTOR) and phosphatase and tensin homolog deleted on chromosome10 (PTEN)/Protein kinase $\mathrm{B}(\mathrm{AKT})$ ) occurred in the cochleae of young mice before major hearing loss. ${ }^{12}$ Treatment with antioxidant corrected pathological molecular changes and endocochlear potential. ${ }^{12}$ In a mouse model of age $\square$ related decline of hearing, the loss of sensory hair cells, spiral ganglion neurons and/or stria vascularis degeneration in the cochlea were associated with low expression of antioxidants and dysregulated reactive species, and Wnt signalling and mitochondrial molecular transport-regulator gene expressions in the cochlear tissues. ${ }^{13}$ In an animal model of noise-induced hearing loss, systemic administration of the water-soluble coenzyme $Q(\mathrm{Co}-\mathrm{Q})$ analogue reduced oxidative-induced cochlear damage, hearing loss and cortical dendritic injury and increased the cochlear levels of Co-Q. ${ }^{14}$ Increased oxidative stress and inflammation may trigger cell death pathways in the cochlea. ${ }^{11}$

In a large cohort study, statistically significant associations between long-term serum C-reactive protein levels and incident hearing impairment were observed in adults aged $<60$ years. ${ }^{33}$ We hypothesise that hyperuricaemia-related inflammation and oxidative stress pathways potentially link gout to the risk of hearing loss in the older adults. These hypotheses need to be tested in future studies which should examine as to which factors contribute to this increased risk and to what extent. Randomised trials of urate-lowering drugs and/or those that reduce oxidative stress independent of urate-lowering are needed to assess their effect on reduction of the risk of hearing loss in older adults.

In conclusion, we found that gout was associated with a higher risk of hearing loss in the elderly, independent of demographic, comorbidities and use of common medications. Future studies should explore whether hyperuricaemia, or inflammation or oxidative stress play a role in this association, and whether treatments that target these pathways can reduce the role of hearing loss in elderly with gout.
Acknowledgements We thank Dr Jeffrey Curtis of the University of Alabama at Birmingham (UAB) Division of Rheumatology who permitted us to reuse the $5 \%$ Medicare data. We thank patients at the UAB gout clinic for asking us the question whether gout is associated with other hearing illness which prompted us to perform this study.

Contributors JAS designed the study, developed study protocol, reviewed analyses and wrote the first draft of the paper. JDC performed the data abstraction and data analyses. All authors made revisions to the manuscript, read and approved the final manuscript.

Funding This material is the result of work supported by research funds from the Division of Rheumatology at the UAB and the resources and use of facilities at the Birmingham VA Medical Center, Birmingham, Alabama, USA.

Disclaimer The funding body did not play any role in design, collection, analysis and interpretation of data; in the writing of the manuscript; and in the decision to submit the manuscript for publication.

Competing interests JAS has received research grants from Takeda and Savient and consultant fees from Savient, Takeda, Regeneron, Merz, Iroko, Bioiberica, Fidia, Crealta/Horizon and Allergan pharmaceuticals, WebMD, UBM LLC, National Institute of Health and the American College of Rheumatology. JAS serves as the principal investigator for an investigator-initiated study funded by Horizon pharmaceuticals through a grant to DINORA, Inc., a 501 (c)(3) entity. JAS is a member of the executive of OMERACT, an organisation that develops outcome measures in rheumatology and receives arms-length funding from 36 companies; a member of the American College of Rheumatology's (ACR) Annual Meeting Planning Committee; Chair of the ACR Meet-the-Professor, Workshop and Study Group Subcommittee; and a member of the Veterans Affairs Rheumatology Field Advisory Committee. JAS is the editor and Director of the UAB Cochrane Musculoskeletal Group Satellite Center on Network Meta-analysis. JDC has no relevant financial conflicts.

Patient consent Not required.

Ethics approval The University of Alabama at Birmingham's Institutional Review Board approved this study, and all investigations were conducted in conformity with ethical principles of research.

Provenance and peer review Not commissioned; externally peer reviewed.

Data sharing statement These data can be obtained from the Centers for Medicare and Medicaid Services (CMS) Chronic Condition Data Warehouse. Data requests can be made to the following email address: (datauseagreement@cms. hhs.gov). The DUA agreement with CMS does not allow us to share these data due to the inclusion of PHI and PII data and the rules of use of Medicare data. We are ready to share the data with colleagues, after obtaining appropriate permissions from CMS Chronic Condition Data Warehouse and the University of Alabama at Birmingham Ethics Committee, related to HIPAA and Privacy policies. Additional information about these data can be found at https://www.resdac.org/resconnect/ articles/148

Open access This is an open access article distributed in accordance with the Creative Commons Attribution Non Commercial (CC BY-NC 4.0) license, which permits others to distribute, remix, adapt, build upon this work non-commercially, and license their derivative works on different terms, provided the original work is properly cited, appropriate credit is given, any changes made indicated, and the use is non-commercial. See: http:// creativecommons.org/licenses/by-nc/4.0/.

\section{REFERENCES}

1. Lin FR, Thorpe R, Gordon-Salant S, et al. Hearing loss prevalence and risk factors among older adults in the United States. J Gerontol A Biol Sci Med Sci 2011;66:582-90.

2. Cruickshanks KJ, Wiley TL, Tweed TS, et al. Prevalence of hearing loss in older adults in Beaver Dam, Wisconsin. The Epidemiology of Hearing Loss Study. Am J Epidemiol 1998;148:879-86.

3. Cruickshanks KJ, Tweed TS, Wiley TL, et al. The 5-year incidence and progression of hearing loss: the epidemiology of hearing loss study. Arch Otolaryngol Head Neck Surg 2003;129:1041-6.

4. Dalton DS, Cruickshanks KJ, Klein BE, et al. The impact of hearing loss on quality of life in older adults. Gerontologist 2003;43:661-8.

5. Bess FH, Lichtenstein MJ, Logan SA, et al. Hearing impairment as a determinant of function in the elderly. J Am Geriatr Soc 1989;37:123-8. 
6. Cacciatore F, Napoli C, Abete P, et al. Quality of life determinants and hearing function in an elderly population: Osservatorio Geriatrico Campano Study Group. Gerontology 1999;45:323-8.

7. Lin FR, Metter EJ, O'Brien RJ, et al. Hearing loss and incident dementia. Arch Neurol 2011;68:214-20.

8. Lin HC, Chao PZ, Lee HC. Sudden sensorineural hearing loss increases the risk of stroke: a 5-year follow-up study. Stroke 2008;39:2744-8.

9. Agrawal Y, Platz EA, Niparko JK. Prevalence of hearing loss and differences by demographic characteristics among US adults: data from the National Health and Nutrition Examination Survey, 19992004. Arch Intern Med 2008:168:1522-30.

10. Kakarlapudi V, Sawyer R, Staecker H. The effect of diabetes on sensorineural hearing loss. Otol Neurotol 2003;24:382-6.

11. Menardo J, Tang Y, Ladrech S, et al. Oxidative stress, inflammation, and autophagic stress as the key mechanisms of premature agerelated hearing loss in SAMP8 mouse Cochlea. Antioxid Redox Signal 2012;16:263-74.

12. Tan WJT, Song L, Graham M, et al. Novel Role of the Mitochondria Protein Fus1 in Protection from Premature Hearing Loss via Regulation of Oxidative Stress and Nutrient and Energy Sensing Pathways in the Inner Ear. Antioxid Redox Signal 2017;27:489-509.

13. Kwon DN, Park WJ, Choi YJ, et al. Oxidative stress and ROS metabolism via down-regulation of sirtuin 3 expression in Cmah-null mice affect hearing loss. Aging 2015;7:579-94.

14. Fetoni AR, De Bartolo P, Eramo SL, et al. Noise-induced hearing loss $(\mathrm{NIHL})$ as a target of oxidative stress-mediated damage: cochlear and cortical responses after an increase in antioxidant defense. $J$ Neurosci 2013;33:4011-23.

15. Martinon F, Pétrilli V, Mayor A, et al. Gout-associated uric acid crystals activate the NALP3 inflammasome. Nature 2006;440:237-41.

16. Cronstein BN, Terkeltaub R. The inflammatory process of gout and its treatment. Arthritis Res Ther 2006;8(Suppl 1):S3

17. Kang DH, Park SK, Lee IK, et al. Uric acid-induced C-reactive protein expression: implication on cell proliferation and nitric oxide production of human vascular cells. J Am Soc Nephrol 2005;16:3553-62.

18. Acharya C, Sharma A, Kantharia N. Involvement of oxidative stress in patients of gout and antioxidant effect of allopurinol. Int $\mathrm{J} \mathrm{Med} \mathrm{Sci}$ Public Health 2015;4:168-72.
19. Glantzounis GK, Tsimoyiannis EC, Kappas AM, et al. Uric acid and oxidative stress. Curr Pharm Des 2005;11:4145-51.

20. Krishnan E. Inflammation, oxidative stress and lipids: the risk triad for atherosclerosis in gout. Rheumatology 2010:49:1229-38.

21. Zamudio-Cuevas Y, Martínez-Flores K, Fernández-Torres J, et al. Monosodium urate crystals induce oxidative stress in human synoviocytes. Arthritis Res Ther 2016:18:117.

22. Singh JA, Hodges JS, Toscano JP, et al. Quality of care for gout in the US needs improvement. Arthritis Rheum 2007;57:822-9.

23. Sudore RL, Karter AJ, Huang ES, et al. Symptom burden of adults with type 2 diabetes across the disease course: diabetes \& aging study. J Gen Intern Med 2012;27:1674-81.

24. Romano PS, Roos LL, Jollis JG. Adapting a clinical comorbidity index for use with ICD-9-CM administrative data: differing perspectives. J Clin Epidemiol 1993;46:1075-9.

25. Moon IJ, Byun H, Woo SY, et al. Factors Associated With Agerelated Hearing Impairment: A Retrospective Cohort Study. Medicine 2015;94:e1846.

26. Braun EM, Karpf EF, Bachna-Rotter S, et al. Conductive Hearing Loss in Association With Urate Deposits in the Middle Ear. Otol Neurotol 2016;37:e252-3.

27. Brown CS, Emmett SD, Robler SK, et al. Global Hearing Loss Prevention. Otolaryngol Clin North Am 2018;51:575-92.

28. Peracino A, Pecorelli S. The Epidemiology of Cognitive Impairment in the Aging Population: Implications for Hearing Loss. Audiol Neurootol 2016;21(Suppl 1):3-9.

29. Fausti SA, Wilmington DJ, Helt PV, et al. Hearing health and care: the need for improved hearing loss prevention and hearing conservation practices. J Rehabil Res Dev 2005;42(4 Suppl 2):45-62.

30. Fukui T, Maruyama M, Yamauchi K, et al. Effects of Febuxostat on Oxidative Stress. Clin Ther 2015;37:1396-401.

31. Dalbeth N, Haskard DO. Mechanisms of inflammation in gout. Rheumatology 2005;44:1090-6.

32. Kalinec GM, Lomberk G, Urrutia RA, et al. Resolution of Cochlear Inflammation: Novel Target for Preventing or Ameliorating Drug-, Noise- and Age-related Hearing Loss. Front Cell Neurosci 2017;11:192.

33. Nash SD, Cruickshanks KJ, Zhan W, et al. Long-term assessment of systemic inflammation and the cumulative incidence of age-related hearing impairment in the epidemiology of hearing loss study. J Gerontol A Biol Sci Med Sci 2014;69:207-14 